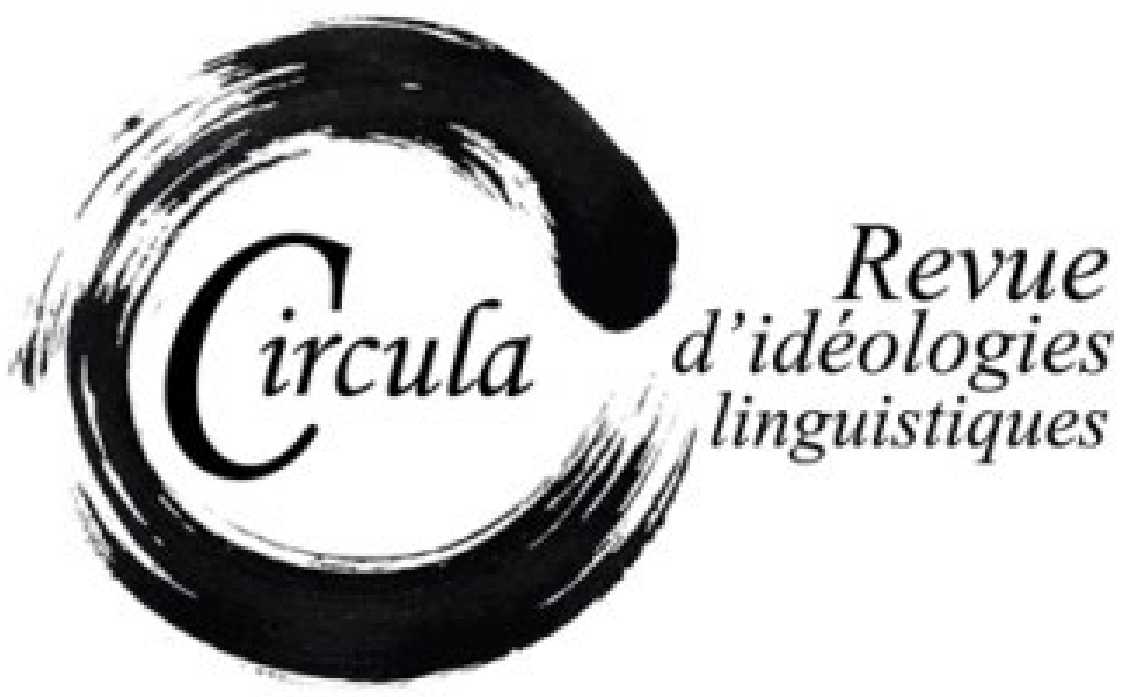

TITRE: LANGUE ROMANE OU ROMANDE ? VARIÉTÉ AUTONOME OU BRIBE DE CONTINUUM ? UN SIÈCLE DE CONSTRUCTION DU FRANCOPROVENÇAL DANS LA GAZETTE DE LAUSANNE (1875-1988)

Auteur(s): Manuel Meune, Université DE MontréAL

Revue: CirCula, NUMÉRO 4

PAGES: $22-42$

ISSN: 2369-6761

DiRECTEURS: WIM REMYSEN ET SABINE SCHWARZE

URI: HTTP://HDL.HANDLE.NET/11143/10175

DOI: $10.17118 / 11143 / 10175$ 


\title{
Langue romane ou romande? Variété autonome ou bribe de continuum ? Un siècle de construction du francoprovençal dans la Gazette de Lausanne (1875-1988)
}

\author{
Manuel Meune, Université de Montréal \\ manuel.meune@ umontreal.ca
}

Résumé : Le francoprovençal ne compte plus de jeunes locuteurs natifs en France (région Rhône-Alpes), mais on en trouve en Italie (Val d'Aoste) et en Suisse (Valais). Cette langue née au Vle siècle et identifiée au XIX', parfois littéraire mais jamais administrative, souvent appelée patois, jouit d'un faible prestige. Elle fait cependant l'objet d'un discours collectif qui mérite l'analyse, s'agissant de ses liens avec le provençal, le français ou l'allemand, de sa disparition ou de sa revitalisation. Pour étudier ses représentations en Suisse romande, nous nous appuyons sur les textes de la Gazette de Lausanne dans lesquels apparaît le terme franco-provençal, de 1875 à 1988 (première et dernière occurrences). Ce terme (accompagné ou non de patois) contribue à la construction de diverses communautés imaginées, associées à la patrie (cantonale ou nationale), à la communauté linguistique francophone (face aux germanophones majoritaires) ou à une entité transnationale regroupant des régions suisses, françaises et italiennes.

Mots-clés : Suisse ; francoprovençal ; patois ; revitalisation ; représentations linguistiques ; identité

Abstract: There are no more young native speakers of Francoprovençal in France (Rhône-Alpes), but they can be found in Italy (Aosta Valley) and Switzerland (Valais). This language, born in the 6th century and identified in the 19th, often called patois, has a low prestige since it was sometimes a literary language but never an administrative one. However, it is subject to a collective discourse that deserves further study, with regard to its links with Provençal, French or German, to its extinction or its revitalization. Our study of its representations in French-speaking Switzerland is based on the texts of the Gazette de Lausanne in which the term franco-provençal appears, from 1875 to 1988 (first and last occurrences). This term (accompanied or not by patois) contributes to the construction of various imagined communities, associated with the 'fatherland' (whether cantonal or national), with the French-language community (facing a majority of German speakers) or with a transnational entity encompassing Swiss, French and Italian regions.

Keywords: Switzerland; Francoprovençal; Patois; revitalization; language representations; identity 


\section{Introduction}

Le francoprovençal (FP) est une langue romane distincte née vers le VIe siècle de la romanisation de la Gaule à partir de Lyon, mais aussi, plus globalement, dans les zones de rayonnement des axes de transit romains qu'étaient les grands cols de l'arc alpin occidental (Grand-Saint-Bernard, Petit-Saint-Bernard, Mont-Cenis, etc., v. Kristol, 2016). Souvent négligé des romanistes eux-mêmes parce qu'identifié au XIX siècle seulement (Tuaillon, 1983), le FP a été historiquement parlé dans le centre-est de la France (surtout le nord de la région Rhône-Alpes), dans l'actuelle Suisse francophone (hormis le canton du Jura), et dans le nord-est de l'Italie (Val d'Aoste et quelques vallées du Piémont). Utilisée à des fins littéraires dès le XIII ${ }^{\text {e }}$ iècle, mais jamais langue administrative, cette langue menacée de disparition est encore un peu pratiquée en France (Savoie, Bresse, Lyonnais), par quelques milliers de personnes âgées et une poignée de néo-locuteurs (Bert, Costa, Martin, 2009). Elle l'est davantage en Italie, où la transmission intergénérationnelle se fait encore à une échelle relativement large. En Suisse romande, où le français domine, des variétés sont encore parlées dans quelques communes des cantons de Fribourg (Meune, 2012b) et du Valais - où le village d'Evolène compte encore de jeunes locuteurs natifs (Matthey, 2012). Mais les parlers vernaculaires ont disparu dès le XIXe siècle des cantons de Neuchâtel, de Genève et de Vaud - sauf dans quelques communes (pour un aperçu de la situation actuelle en Suisse, v. par exemple Matthey, 2012 ; Meune, 2012a).

En Suisse comme ailleurs, le glottonyme savant, franco(-)provençal, est peu utilisé par les locuteurs (Meune, 2012a : 67 ; Meune, 2012b : 33), qui préfèrent le terme patois - bien que souvent connoté négativement chez les non-locuteurs. La Suisse, officiellement quadrilingue (allemand, français, italien, romanche), n’a pas mieux préservé le FP que la France, laquelle y a en partie exporté son idéologie unilingue. Mais si les cantons ont banni la langue ancestrale des écoles, des intellectuels l'ont parfois valorisée en même temps que leur patrie (cantonale ou romande), tandis que des universitaires ou des associations entreprenaient de collecter des données ou de maintenir, voire (plus rarement) de revitaliser la pratique de la langue.

La nation atypique qu'est la Suisse plurilingue est un terrain propice à l'étude de l'évolution des représentations de cette langue atypique qu'est le FP. Nous nous pencherons ici sur la Gazette de Lausanne, fondée en 1798, qui a fusionné deux siècles plus tard (1998) avec le Journal de Genève pour donner naissance à l'actuel quotidien de référence romand, Le temps. Grâce aux 37 textes obtenus par le biais d'archives en ligne ${ }^{1}$ à l'aide du mot-clé franco-provençal pour la période 1875-1988 (dates des première et dernière occurrences du mot)², nous analyserons les perceptions du FP, en observant la façon dont ces textes illustrent divers types de construction linguistique et identitaire, vaudois,

1. www.letempsarchives.ch

2. À moins qu'elle ne soit liée à une erreur de type technique sur le site d'archivage, l'absence d'occurrences entre 1988 et 1998 (année de la disparition du journal) semble en soi révélatrice d'un contexte sociolinguistique où la question de la langue autochtone de Suisse romande, considérée comme secondaire, ne faisait guère l'objet, à la fin du siècle dernier, de représentations collectives en mouvement. 
romand ou, plus rarement, FP (transnational). Ces textes contiennent au moins une occurrence du mot franco-provençal, comme substantif et adjectif, y compris avec les marques du féminin et/ou du pluriel (-çale/s/-çaux). Ils peuvent aussi contenir des références aux mots patois, dialecte ou parler, mais le corpus n'inclut pas les textes (trop nombreux pour être traités ici) qui comprendraient des éléments relatifs au FP sans référence explicite au concept.

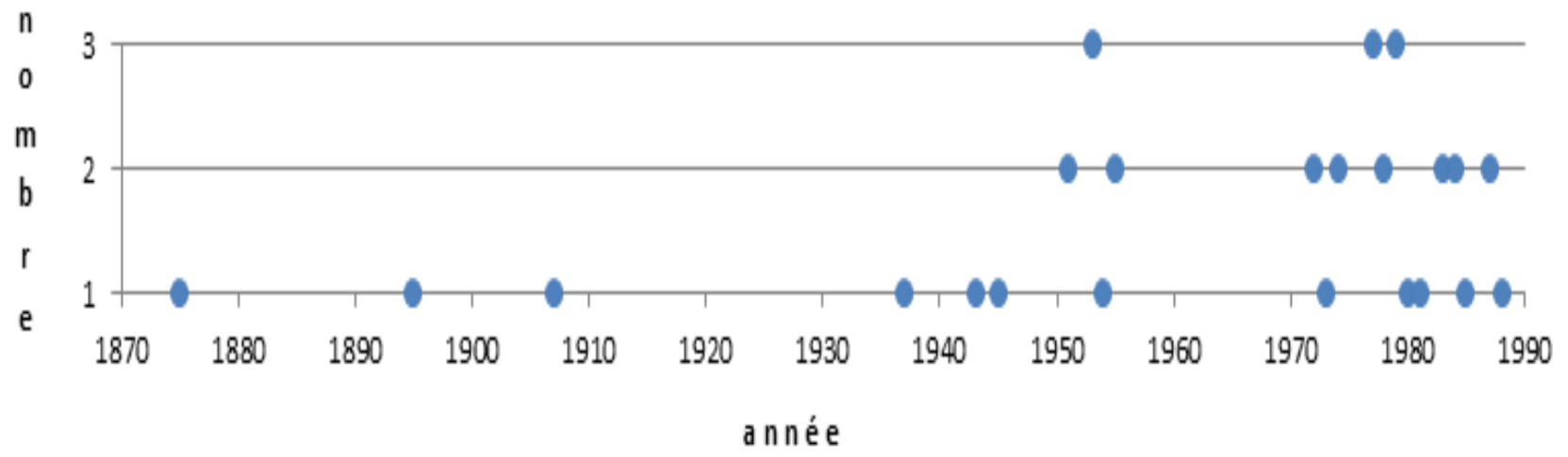

Graphique 1. Textes ayant (au moins) une occurrence pour franco-provençal (1875-1988)

Le glottonyme franco(-)provençal, loin d'avoir supplanté le terme patois, réoriente quelque peu la construction des communautés imaginées (Anderson, 1991) et rend possible un discours protonational (Hobsbawm, 1992), par exemple en remplaçant les affiliations locales ou nationales par une appartenance transfrontière incluant le Val d'Aoste ou la Savoie - nous verrons toutefois que cette dernière reste largement absente du corpus, contrairement à un corpus de textes du Journal de Genève que nous évoquerons brièvement. Signalons que les auteurs des textes, lorsque ceux-ci sont signés, sont parfois des journalistes de la Gazette, mais plus souvent des spécialistes (linguistes, écrivains, universitaires) sur lesquels nous donnerons quelques informations lorsque nous le pourrons. La nature et la longueur des textes varient. Il peut s'agir de courtes dépêches d'agence ou de notices (à l'occasion de décès), de communiqués (pour annoncer une conférence), de lettres de lecteurs, mais aussi, dans une plus grande proportion, d'articles de fond relevant des pages littéraires et culturelles. Nous nous concentrerons particulièrement sur certains de ces textes-clés qui permettent d'illustrer l'éventail des représentations linguistiques en présence ${ }^{3}$.

3. Nous sommes conscient que le discours médiatique que produisent des acteurs représentant largement le monde littéraire et universitaire n'est pas a priori plus légitime et emblématique de la conscience collective et qu'il importerait, dans une étude complémentaire, de mettre en regard leurs représentations avec celles qu'on observe dans d'autres segments de population - patoisants ou non. 


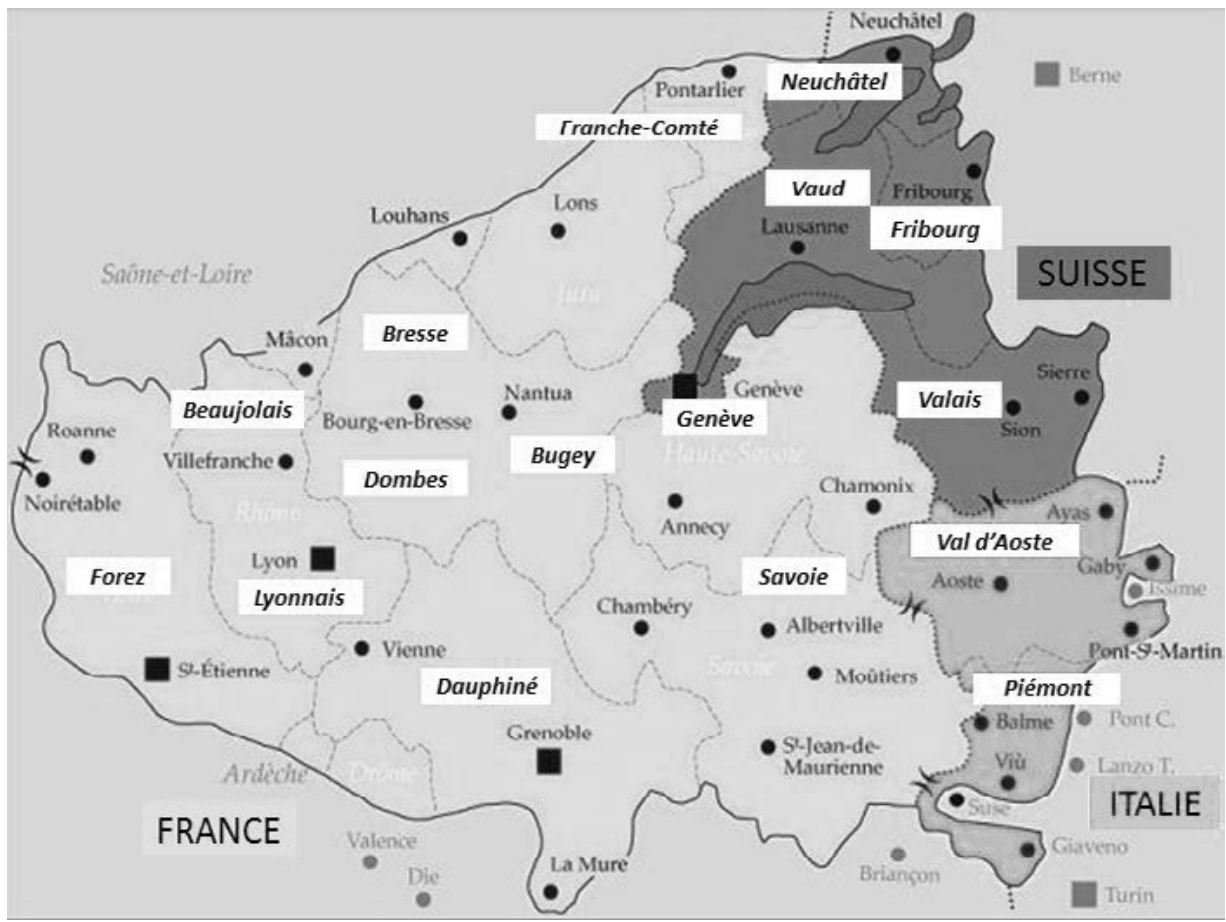

Illustration 1. Les principales villes et régions du domaine francoprovençal (d'après une carte du Centre d'études francoprovençales, Saint-Nicolas, Val d'Aoste)

\section{L’apparition du francoprovençal sur fond de mode provençaliste}

La première trace du FP dans la Gazette de Lausanne apparaît dans un article sur un congrès de romanistes à Montpellier (1875.10.4). Dans ce compte-rendu non signé, on apprend que le prix de philologie a été accordé à Grazialdio Isaia Ascoli, professeur à Milan, pour ses Schizzi franco-provenzali - qui marquent traditionnellement l'acte de naissance du FP comme langue romane autonome. Dans la version définitive du texte (1878), Ascoli y désigne le FP comme « un type linguistique » qui réunit des caractères dont certains sont communs avec « le provençal » (pris dans le sens large de langue d'oc) et d'autres avec «le français ». Il précise que ce type «ne provient pas d'une tardive confluence d'éléments divers », mais dispose de «sa propre indépendance historique », peu différente de celle des « autres principaux types romans ». Malgré cela, l'ambiguïté attachée au concept même de franco(-)provençal (langue autonome ou mélange ?) n'est pas levée et persistera jusqu'à nos jours (v. plus bas). Dans l'article cité, Ascoli lui-même, qui vient de révolutionner la bipartition traditionnelle de l'espace gallo-roman (avec deux grands domaines, oïl et oc), hésite à officialiser la naissance d'une troisième entité : dans le télégramme d'amitié qu'il adresse aux congressistes, il se contente de clamer, de façon très consensuelle, « Viva la Francia d'oc, viva la Francia d'oïl ! ». Du reste, le rythme du congrès n'étant guère bouleversé par le message, c'est surtout le provençal qui attire l'attention du journal suisse, en particulier son regain de prestige et le renouveau que lui prédit l'écrivain Frédéric Mistral en dépit des signes de déclin. 
Si l'approche mistralienne ne concerne pas directement le domaine FP, elle fournit un point de comparaison à la presse romande (v. aussi Meune, à paraître), lorsqu'il s'agira d'ébaucher une politique linguistique pour le FP et de concilier plurilinguisme et patriotisme. L'article de 1875 évoque ainsi la Révolution française dont « les législateurs ne dédaignaient pas de parler au peuple en sa langue » - provençal, breton ou alsacien - à une époque où on pouvait « aimer la France » sans devoir « balbutier français », avant que l'approche jacobine unificatrice de l'abbé Grégoire ne l'emporte. Cette thématique trouve un écho un siècle plus tard, lorsque dans un long article sur lequel nous reviendrons à quelques reprises (1979.7.7), l'essayiste et journaliste Jean-Philippe Chenaux décrit une Révolution vaudoise qui, «suivant l'exemple de Paris », voit dans le parler régional « un vestige humiliant de l'Ancien Régime », ce qui amène le canton, en 1806, à décréter que «[l]es Régens [instituteurs] interdiront à leurs écoliers, et s'interdiront absolument à eux-mêmes, l'usage du patois, dans les heures de l'École » - ce fut le cas plus tard dans d'autres cantons, notamment Fribourg (v. Gadient, 2012). Même si, en 1806, le terme franco-provençal ne pouvait être utilisé, le rappel de la «violence » du combat contre le « parler régional » en terre vaudoise permet de donner au FP un surcroît de dignité en rappelant qu'il a été combattu au même titre que d'autres langues plus reconnues - comme le provençal.

Si la référence régulière au provençal permet d'aborder des considérations (socio)linguistiques, elle entretient certaines confusions. On trouve la deuxième occurrence de franco-provençal dans une lettre du philologue lausannois Jean Bonnard à la rédaction (1895.7.22), qui traite de l'absence d'influence sur l'article défini du $h$ des mots d'origine germanique, dans les domaines tant FP qu'occitan. Le fait que dans le pays de Vaud, on dit l'halle et l'hache (et non la halle et la hache) est l'occasion d'expliquer que les parlers vaudois sont apparentés au français et au provençal, puisque le canton fait partie « du domaine franco-provençal, ce qui revient à dire que le langage vaudois et le provençal ont plusieurs caractères communs ». Dans cette phrase comme ailleurs dans l'article, l'auteur semble insister moins sur le français que sur le provençal, ce qui, en plein engouement pour le mouvement félibrige (v. Meune, à paraître), confère aux parlers romands un prestige par association, mais ne permet guère de décrire toute leur originalité.

On trouve un exemple plus marqué d'ambiguité dans un article où Edmond Jaloux, académicien d'origine marseillaise qui a vécu en Suisse, établit un lien étroit entre le provençal et le FP, ce dernier semblant inclus dans la mouvance provençale (1945.6.9) : dans un premier temps, il constate que la langue d'oc est distribuée en dialectes limousins, gascons, languedociens, auvergnats, dauphinois et provençaux, mais il ajoute immédiatement que « [c]ertains des dialectes de la Suisse romande ont formé même [nous soulignons] un groupe linguistique qu'un savant, M. Ascoli, a baptisé le franco-provençal ». Ici, c'est l'idée du continuum linguistique qui semble dominer plutôt que celle de l'existence de trois grandes entités romanes en France. Et l'auteur ajoute quelques réflexions sur le continuum que constitue toute langue, évoquant le caractère « assez mouvant » de la question, puisque « sur les origines des langages romans, l'opinion des savants est extrêmement variable ». Ce tropisme provençal apparaît aussi dans l'hommage rendu à André Desponds (1983.8.16), présenté 
comme un érudit du FP, « qui fut la langue d'une grande partie des pays du Midi de la France [nous soulignons] et du pays romand actuel ». Car même les territoires les plus méridionaux de l'espace FP en France (région de Grenoble, sud de la Savoie) ne font pas partie de ce qu'on associe au Midi. Quant à l'interview d'Emmanuel Le Roy Ladurie (1980.12.27), elle ne contribue guère à préciser les contours du domaine FP, car s'agissant de la Suisse romande, l'historien français semble hésiter entre ses ancrages méridional (au sens large, presque mythique) et franco-provençal (plus restreint) : « Je vous rappelle que le dialecte franco-provençal était le dialecte des Romands également. J'ai une espèce d'intérêt pour la France du sud, dans laquelle j'inclus Genève ». On voit ainsi que même vers la fin du $20^{e}$ siècle, la nature des liens du FP avec la méridionalité ne semble pas toujours claire - elle ne l'est d'ailleurs pas plus dans certaines œuvres littéraires (v. Meune, 2015).

\section{Un « groupe franco-provençal » aux frontières approximatives}

C'est souvent à propos de linguistique que le terme franco-provençal est employé. Comme substantif, il est parfois complété par un adjectif ou une définition (« le franco-provençal, cette langue que l'on parle de l'Isère au nord du Piémont », 1988.2.27). II peut être complément de nom : on évoque son « aire linguistique » (1972.4.28), sa « région » (1974.8.15), son « nom » (1907.1.24), ses « origines » (1979.7.7), sa «formation» (1979.7.7), ses « connaisseurs 》 (1983.8.16). Comme adjectif, il qualifie des termes comme « domaine » (1895.7.22), « phonétique » (1937.4.25), « souche » (1974.8.15), « littérature » (1979.3. 31). Dans les cooccurrences, le terme patois est assez fréquent (« écrire en vieux patois franco-provençal », 1978.9.30; « [recul des] patois franco-provençaux », 1954.9.24). Mais ces patois sont aussi appelés parlers ou dialectes, certains universitaires préconisant des termes neutres : il est alors fait référence aux frontières des « parlers franco-provençaux » (1979.7.7), à la prise en compte des « dialectes franco-provençaux » dans la toponymie (1985.2.11), etc. Au plus tard dans les années 1970 et 1980, le terme franco-provençal semble s'être implanté, malgré l'attachement à patois. Et la Radio suisse romande, qui a joué un rôle important dans la promotion des parlers locaux, utilise parfois le terme franco-provençal pour annoncer des émissions (v. par ex. 1988.2.27).

Dans la Gazette de Lausanne, il est souvent question du « groupe franco-provençal » sans que cela permette de préciser les contours de la zone FP. Certes, le concept apparaît parfois neutre et compatible avec l'idée d'une langue distincte (v. cet article sur le francoprovençaliste Louis Gauchat: 1937.4.25), et les délimitations peuvent être précises, comme dans un texte sur le Glossaire des patois de la Suisse romande (GPSR), où l'on fait bien la différence entre les parlers romands qui, dans leur majorité, font partie du « groupe franco-provençal » et le parler jurassien, qui relève de la « langue d'oïl » (1972.2.1). Il arrive que des détails soient donnés pour expliquer ce qui apparente le FP au provençal et au français, s'agissant par exemple du $a$ tonique resté $a$ comme en provençal, mais devenu $i$ devant une palatale comme en français, à l'instar de portâ (porter / prov. portar) et tserdzi (charger / ancien fr. chargier) (1979.7.7). En outre, l'auteur, Chenaux, rejette ici les théories sur l'« héritage des Burgondes » venus du Danemark et sur la coïncidence entre les frontières du premier Royaume de Bourgogne et celles du domaine FP (dont la langue germanique aurait influencé la langue romane 
du lieu). II rappelle que le Val d'Aoste, région FP s'il en est, n'a jamais été habité par les Burgondes, ce qui confirme la thèse d'une langue distincte antérieure.

Pourtant, dans nombre d'articles, les choses sont moins claires. Dans un texte non signé sur le GPSR (1973.1.13), le concept de groupe alimente le flou concernant les limites du FP: ce dernier, dont le « groupe d'oc » serait proche, est intégré à la mouvance occitane: « les deux groupes de patois romands, l'un d'oïl, comprenant les dialectes du Jura et du canton de Neuchâtel, l'autre d'oc, apparenté déjà au franco-provençal [nous soulignons], avec les patois du Pays de Vaud, de Fribourg, du Valais et de Genève ». De plus, le canton de Neuchâtel - situé en zone FP - est associé à la zone d'oïl. Autre exemple d'approximation : un texte sur la Fête des patois romands qui se tient à Mézières et au Château d'Oron (Vaud). Dans la description que fait de cette « grande fête du vieux parler » Charles Montandon, journaliste radiophonique impliqué dans la sauvegarde du FP, on lit que les patois du «nord du Jura » appartiendraient eux aussi au « groupe franco-provençal» (1977.8.29).

S'agissant de la zone FP à l'extérieur de la Suisse, on remarque également que diverses régions lui sont attribuées à tort. Ainsi le patois vaudois « appartient au groupe franco-provençal au même titre que les dialectes de la Bourgogne [nous soulignons], de la Savoie, de la Vallée d'Aoste, du Lyonnais, du Dauphiné, de l'Auvergne [n.s.] » (1978.3.16). Si une petite partie de la Bourgogne (Bresse louhannaise) est dans le domaine FP, ce n'est pas vrai de l'Auvergne. Et dans son long article, Chenaux, qui donne ailleurs des informations précises, présente le vaudois comme un dialecte appartenant « au groupe dit "franco-provençal" qui fait la transition [nous soulignons] [...] entre les anciennes langues d'oc (midi de la France) et d'oïl (nord de la France) » (1979.7.7). Non seulement on ne sait donc plus si le FP est une langue distincte ou un élément de continuum, mais le « groupe franco-provençal » comprend selon lui, outre le valdôtain, le savoyard et le lyonnais, également «le mâconnais ». Or, cette dernière référence surprend puisque la région de Mâcon fait partie du domaine FP de façon plutôt marginale, avec un parler très francisé relevant moins clairement du FP et ayant produit moins d'écrits littéraires que, par exemple, le bressan voisin - très peu évoqué dans la Gazette de Lausanne.

Parfois, au-delà du flou des limites du domaine FP, l'existence même d'une langue distincte est mise en cause. Dans un texte sur la minorité de langue roumaine en Istrie (1955.7.23), l'universitaire Aldo Dami évoque ainsi des « groupes de dialectes comme le wallon ou le franco-provençal (qu'il convient de rattacher au français [nous soulignons]) », et le FP n'est pas au nombre des neuf langues romanes énumérées (portugais, espagnol, catalan, provençal, français, rhéto-romanche, sarde, italien, roumain).

Malgré les approximations, la connaissance du fait FP croît peu à peu, et ce dernier sert parfois à justifier une coopération transfrontalière, par exemple à propos de la « région lémano-alpine ». Bien que les facteurs d'unité soient présentés comme étant " potentiels ", l'existence de cette région est postulée par l'Institut universitaire d'études européennes - piloté par Denis de Rougemont. Ainsi, cette région recouvrirait « à peu près l'ancien royaume de Bourgogne et l'aire linguistique du franco-provençal » (1972.4.28). La question linguistique passe alors au second plan, de même que, 
quelques années plus tard, lorsqu'il est question du « Grand Delta », région française allant de Lyon aux frontières espagnole et italienne : elle présenterait de profondes analogies avec la Suisse romande, elle-même héritière de la « civilisation franco-provençale » (1977.8.5). Ce dernier concept est vague, mais son utilisateur a sans doute entendu le terme franco-provençal dans un contexte lié à la langue et, induit en erreur par son caractère hybride, l'a élargi à toute une « civilisation »; ses contours sont imprécis, mais cela n'en montre pas moins que l'idée FP circule et qu'elle peut susciter la rêverie identitaire.

\section{Entre discours sur la langue et discours sur la (petite) patrie}

Si la connaissance des limites territoriales du FP n'est pas anodine, c'est aussi parce que le caractère transfrontalier du FP entre parfois en concurrence avec la construction identitaire romande. Le corpus n'offre que deux exemples d'association du terme franco-provençal avec le possessif notre, ce qui témoigne tout au plus d'une ébauche de construction d'un nous FP, d'une adaptation au discours identitaire généralement associé au patois: « notre franco-provençal [est apparenté au latin des Alpes] » (1953.12.26) ; « enseigner notre franco-provençal » (1955.3.30). Sinon, en matière de langue, la construction identitaire s’articule autour de références liées à la Suisse, plutôt que transnationales.

Au début du 20e siècle, le philologue vaudois Ernest Muret (1907.1.24) insiste sur la dignité de « nos patois », qui ne sont pas davantage « du sale latin » que le " patois de l'île de France ». II rappelle qu'ils font partie du FP, mais il préfère appeler la langue romane concernée « le romand ». Par métonymie, en désignant la partie pour le tout, il donne l'impression que cette langue est authentiquement suisse (voire plus suisse que française) : « le romand s'intercale entre les dialectes d'oïl et le provençal ». Le discours, au-delà des considérations linguistiques, a des ramifications identitaires. Même s'il s'agit d'apprivoiser le nouveau mot technique (franco-provençal), l'important semble être de faire coïncider discours sur la langue et discours sur la patrie ; le patriotisme peut du reste être d’obédience régionale/cantonale (vaudoise) ou nationale/infranationale (suisse/romande), les deux formes n'étant nullement contradictoires et se nourrissant l'une l'autre (v. Meune, à paraître).

Même au milieu du siècle, le tropisme helvétique reste sensible. Ainsi, dans un article sur les langues romanes des Alpes (1953.12.26), Aldo Dami fait preuve d'un patriotisme suisse consistant à afficher la fierté pour la diversité des langues autochtones de Suisse - y compris le FP. Lorsqu'il évoque la parenté entre «notre romanche » avec «notre franco-provençal », il opère une helvétisation du fait FP. Cette dernière passe par l'insistance sur les Alpes - marqueur identitaire essentiel - tout au long de l'article, mais aussi par le rapprochement avec le romanche - icône du plurilinguisme - devenu en 1938 langue nationale. Notons que lorsque l'auteur fait du romanche et du FP les descendants d'un ancêtre commun, le "latin des Alpes », cette alpisation ou helvétisation symbolique s'éloigne des théories des linguistes, pour qui les deux langues ne sont pas directement liées, et qui rappelleraient que le FP, diffusé à partir de Lyon, n'avait aucun lien avec l'ancienne Rhétie romaine. 
Quelquefois, il y a moins helvétisation que vaudisation. Un article insiste ainsi sur l'autonomisation du patois vaudois qui se serait « détaché par transitions insensibles du tronc originel » (1907.12.24). Cette glottogenèse va à l'encontre des théories de ceux qui contestent l'idée d'un tronc commun FP et préfèrent parler d'une naissance conjointe de plusieurs variétés (sans remettre en cause l'unité du domaine, v. Greub, 2012), mais elle permet d'individualiser la petite patrie vaudoise. Dans un article ultérieur (1983.3.2), le canton devient même le cœur de la zone FP, dans un bel exemple de vaudo-centrisme. À en croire le journal, pour le linguiste Maurice Bossard, le canton de Vaud serait le foyer originel du domaine FP, un lieu dont la langue aurait peu à peu gagné les territoires environnants: "Ce patois n'est d'ailleurs pas exclusivement vaudois [nous soulignons] dans la mesure où il a rayonné [n.s.] au-delà des frontières cantonales, jusqu'en [n.s.] Savoie, dans le Lyonnais, la Franche-Comté et le Dauphiné ». S'il n'est pas exclu que les propos aient été simplifiés ou déformés par la rédaction, ce qui est à l'œuvre est une inversion de l'histoire puisque, rappelons-le, c'est de l'ancienne Lugdunum qu'est partie la romanisation de la région.

Si le nous semble plus facile à accoler à patois qu'à franco-provençal, c'est que l'énumération des régions du domaine FP, inévitable puisque les faits linguistiques sont peu connus, impose aux rédacteurs un ton didactique ne favorisant guère l'identification collective, alors que le mot patois permet de mêler aisément langue et origine (mythifiée) : « nos patois [nous soulignons], descendants directs du bas-latin que parlaient nos ancêtres [n.s.] gallo-romans et restés souvent plus proches du latin que le français » (1981.8.29). Par ailleurs, la construction d'un nous patois transcende parfois le cadre du FP, comme en témoigne le titre d'une émission de la Radio romande : « Nos patois : présentation et comparaison des patois wallon, jurassien et franco-provençal » (1984.11.10). La mention du FP ne contredit pas l'idée d'une entité FP distincte des parlers d'oïl (jurassien et wallon), mais elle fait aussi écho à un nous qui dépasse la communauté des francoprovençalophones pour s'adresser à celle des patoisants - car quelle que soit l'origine des patois, parler une langue non normée constitue une expérience particulière qui, comme telle, peut faire l'objet d'un discours à forte saveur identitaire - ce qui explique aussi pourquoi les locuteurs de parlers locaux tiennent au mot patois.

\section{Une conscience parcellaire de la diversité littéraire du FP}

Si la conscience d'une unité de l'aire FP fait défaut, c'est aussi qu'il n'existe aucune littérature canonique, diffusée dans l'ensemble du domaine. Un article évoquant les « longs siècles [où] nos patois n’ont été qu'une langue parlée » (1907.1.24) souligne la (prétendue) pauvreté du corpus littéraire dans la zone FP. Pourtant, l'auteur semble confondre zone FP et zone romande puisqu'il s'en tient à la partie suisse du domaine et n'évoque pas les textes plus anciens et à la qualité littéraire incontestable (venant de Bresse, du Lyonnais ou de Savoie) : soit parce qu'il n'en a pas connaissance (ce qui invite à réfléchir à l'absence historique - explicable - d'un corpus de textes canoniques pour le FP, mais aussi, pour l'avenir, à l'éventuelle transcription dans une orthographe supradialectale lisible d'œuvres écrites selon diverses graphies phonétiques locales, parfois peu compréhensibles à l'extérieur de leur zone de référence), soit parce qu'il juge que cela ne concerne pas son propos (ce qui 
montre le faible intérêt pour la transnationalité du domaine FP). La fierté qui pourrait résulter d'une conscience que l'aire FP a produit ailleurs de grandes œuvres semble alors peu applicable au discours identitaire à l'usage des Suisses, et la construction littéraire d'une communauté imaginée FP transnationale importe moins que le cadre national habituel. Si l'article mentionne que le « premier document patois en Suisse [...], le "Cé qu'è l'en haut" », est encore entonné par les Genevois « autour de la dinde traditionnelle», on se borne à constater que « les patois romands ont pris rang de langue écrite » au XIXe siècle, sans saisir l'occasion de regarder au-delà des frontières.

Jean-Philippe Chenaux fait également valoir que le domaine est « extrêmement pauvre en œuvres littéraires » (1979.7.7), parce que les centres littéraires médiévaux fréquentés par les troubadours n'existaient pas dans le domaine FP qui, de surcroitt, connut peu de guerres (" on n'avait alors pas grand-chose à raconter ! »; v. aussi 1983.3.2). Constatant que « notre patois n’a jamais accédé au rang de langue littéraire », il évoque une dizaine de textes (" autant dire pratiquement rien ») composés en FP en Suisse avant 1630. Mais la représentation du FP reste helvéto-centrée, et malgré quelques références aux autres régions, rien n'incite les Romands à la fibre littéraire à revendiquer pour eux de prestigieux textes venus d'ailleurs - par exemple celui de Marguerite d'Oingt, écrit en lyonnais dès le XIII e siècle, ou des pièces de théâtre en bressan de la fin du XVIe siècle (d'Oingt, 2012 ; Tuaillon, 2001).

Chenaux évoque en outre le paramètre religieux, la disparition rapide du patois dans les cantons protestants où les prédicants d'origine française, après 1536, « ont imposé la langue de Paris » pour lire la Bible (1979.7.7). Il rappelle que ce n'est qu'en 1951 que le pasteur Louis Goumaz a traduit en vaudois certaines paraboles. Par ailleurs, la maigreur du corpus littéraire FP s'expliquerait par le fait que les chants profanes étaient interdits en terre protestante. Et malgré l'apparition de textes en patois vaudois au XVIII siècle (comme Le conto dau craizu, « le conte de la lampe à huile »), la Révolution vaudoise avait freiné l'essor littéraire réel et induit une démarche glottophobe (Blanchet, 2016) consistant, comme en France, à donner un objet (en l'occurrence un bouton) à un enfant surpris à parler patois ; à la fin de la journée, l'enfant avait une punition, à moins qu'à son tour il prenne en défaut un camarade. Cette « délation [...] institutionnalisée », redoutablement efficace, constate l'auteur, a permis d'éradiquer rapidement le vaudois (sauf dans le Jorat et les Préalpes). Certes, rappelle-t-il, des intellectuels s'y sont intéressés (Benjamin Corbaz, Charles César Dénéréaz, Philippe Bridel, Louis Favrat) et ont composé grammaires et glossaires (comme le Petit dictionnaire vaudois publié en 1962 par Albert Chessex) ; la codification de l'orthographe vaudoise par Jules Cordey a été un autre temps fort et on a vu paraitre des textes en patois dans la Feuille d'Avis de Lausanne, ainsi que des traductions de textes-clés comme L'Avare, ou les Trois cloches - mais rien qui, laisse entendre Chenaux, ressemble à un véritable canon d'œuvres littéraires. 


\section{Disparition inéluctable et impossible revitalisation}

Qu'en est-il du discours sur la possibilité de conserver vivants les parlers locaux - qui portent désormais le nom, plus prestigieux, de franco-provençal ? Dans un article emblématique de l'attitude générale (1981.8.29), la position est claire : « nos patois [...] sont en train de mourir dans l'indifférence. [...] Dans quelques décennies, le langage de nos ancêtres romands ne survivra que dans des noms de familles et de lieux, des accents régionaux, des termes de métier, des expressions typiques ». Si la mort du vaudois est inéluctable, c'est qu'il était « la langue d'une civilisation rurale », d'une « société paysanne et artisanale », et qu'il ne peut «survivre dans une civilisation nouvelle ». Aucune trace d'intérêt pour une revitalisation qui passerait par une standardisation ou par la création de néologismes. "Les dialectes de la Suisse romande ne verront pas le XXIe siècle », lit-on encore, faute de masse critique («quelques dizaines de milliers de patoisants, sur un million de Romands »), et faute d’appui hors des cercles de spécialistes («Aujourd'hui, seuls s’y intéressent encore de savants philologues »). Le paradoxe veut que les patois romands n'aient « trouvé leur forme écrite qu'au bord de l'extinction ». Mais si l'auteur évoque un « chant du cygne [...] remarquable » et un théâtre dialectal «florissant», les tentatives pour « réhabiliter le patois » et inverser les effets des politiques linguicides lui paraissent trop tardives : l'effacement se poursuit, « inéluctablement et sans contrainte, par l'évolution démographique, économique et culturelle».

Certaines conceptions illustrent le lien perçu comme indissoluble entre peuple et langue. L'auteur cite Juste Olivier pour qui « [l]a langue d'un peuple est toute son histoire », puisque « le verbe d'un peuple » en est « la substance et l'esprit », qu'il est « le peuple même ». Il évoque l'icône de la littérature romande, Charles Ferdinand Ramuz, qui disait que c'est « au patois qu'il faut tendre à tout ramener », même lorsqu'on est de langue maternelle française, ou encore l'écrivain Jules Cordey, pour qui les Vaudois, en même temps que leur «langue ancestrale », vont « perdre leur âme ». Pourtant, la non-coïncidence entre peuple et ancienne langue n'est pas dramatisée et l'article souligne avec fatalisme, sans amertume, que même avec des cours de patois, « il n’y aura pas de miracle ».

Et dans un article sur le GPSR (1973.1.13), lorsqu'est rappelé que celui-ci visait à « recueillir scientifiquement » des patois dont les créateurs sont « le peuple », c'est l'aspect conservatoire qui est mis en avant, et non le paradigme de la transmission. L'auteur, qui semble vouloir échapper au reproche d'irréalisme, précise ainsi : "les patois, sans qu'on les ressuscite [nous soulignons], méritent qu'on les enregistre ». L'article relève aussi le fait - paradoxal - que la fondation de cercles de patoisants, loin d'être un signe de vitalité, souligne précisément que « le dialecte se meurt lentement » et qu'il est « condamné ». La pratique actuelle du patois n'est pas vue comme une base possible pour revitaliser la langue ; le fait que les « terres traditionalistes et catholiques » du Valais maintiennent encore «l'emploi vivant et quotidien du patois » est associé au fait que ce «langage réaliste, savoureux, cru » serait « admirablement adapté aux besoins d'une population rurale » - le sous-entendu étant, là encore, que la langue disparaîtra en même temps que l'ancien mode de vie rural. 
Dans son article au titre évocateur, « Un parler qui ne veut pas mourir. Chant du cygne du patois vaudois? » (1979.7.7), Chenaux rappelle que le patois vaudois a été « pendant des siècles la langue de tout le peuple vaudois ». Mais malgré l'évocation des « doux regrets de voir disparaître ce savoureux langage que nos aïeux ont oublié d'enseigner à leurs enfants », du « riche vocabulaire autochtone » et des « mots qui ne se retrouvent pas en français », le ton nostalgique ne se transforme pas en discours militant. On trouve certes quelques passages de type performatif où, comme pour renforcer par la parole les conditions d'une éventuelle renaissance, l'auteur feint de croire à cette dernière. Il souligne l'influence de l'Association des patoisants vaudois et du Conseil des patoisants romands, et pour appuyer l'espoir de « redonner vie in extremis » au FP, il cite Gonzague de Reynold, chantre romand des langues du terroir : « un dialecte ne meurt que quand on le laisse mourir » et «il suffit de la volonté de quelques hommes, d'un seul peut-être, pour le ranimer». Et dans « Les Vaudois réapprennent le patois » (1978.3.16), il est question de l'« intérêt croissant pour cette vieille langue » et de l'Université populaire qui donne des cours sur le patois ou de patois - fréquentés par des jeunes, souligne-t-on, pour (se) donner l'illusion que la relève serait assurée. Quant à l'auteur de « Renaissance du patois vaudois?» (1979.3.31), il se félicite du passage de relais entre les locuteurs natifs des « dialectes franco-provençaux de Suisse romande » (de moins en moins nombreux) et les néo-locuteurs (v. Meune, 2012a), encouragé par un Département de l'instruction dont le programme de formation en patois est suivi par 40 enseignants. Néanmoins, cet optimisme de rigueur, qu'on observe dans de rares articles, reste finalement très prudent.

\section{Priorisation du français, vaudoisismes et germanismes, purisme et bilinguisme}

Divers rédacteurs, en plus de tenir pour acquise la disparition du patois, subordonnent clairement la cause du FP à celle du français (régional ou non). Un auteur s'insurge ainsi contre les injonctions du « jacobinisme étroit » venu de France, qui crée chez les écoliers suisses des « complexes d'infériorité », et estime qu'on aurait pu « bâtir sur le patois, en extraire la sève française, les tournures bien vivantes » puisque les pives des conifères ne sont nullement « moins odorantes » que les cônes du français standard (1973.1.13). On comprend alors que les vertus intrinsèques du patois, ne justifiant pas à elles seules la revitalisation de la langue, devraient au moins enrichir le français de Suisse.

À l'occasion de la retraite du linguiste Maurice Bossard, un auteur insiste, comme pour donner des gages de non-ringardise, sur le fait que ce «Vaudois "pure laine" » cultivait le vaudois « sans passéisme », et que l'étude de l'ancienne langue visait surtout à mieux comprendre l'état actuel du français (1983.3.2). Beaucoup, pour légitimer la défense du patois sans se faire taxer de réactionnaires, font donc du zèle dans la défense du français. En jumelant deux démarches, ils contribuent à l'œuvre patriotique suisse, qui implique qu'on soit fidèle aux langues prestigieuses (allemand et français), mais aussi, dans une moindre mesure, aux langues autochtones locales (patois romands et dialectes alémaniques, v. Meune, 2015). Le travail mémoriel d'archivage du FP est vu comme complémentaire de l'attachement à la langue française - dont l'épanouissement est réputé plus utile pour la société: 
Il y a un très grand intérêt à ce que tout nous parlions français, et même [...] le bon français. L'unité morale, la facilité et le plaisir des relations personnelles et générales s'en accroissent. Mais il n'est pas moins urgent de conserver nos patois. Cet acte de piété patriotique ne les sauvera pas de la mort à laquelle ils sont condamnés: du moins subsisteront-ils comme, derrière les vitrines d'un musée [...], les échantillons des espèces animales qui se raréfient et s'éteignent. Il importe que nous sachions ce que et comment ont pensé nos devanciers. (1907.1.24)

On trouve des arguments similaires dans l'article « Le patois langue vivante » de l'écrivain Maurice Zermatten (1955.10.30), qui donne aussi des gages de non-fanatisme en insistant sur les bienfaits du patois pour la santé du français. Si le FP, « langue vivante pour de nombreuses régions de notre Suisse romande », garde "toutes ses raisons de vivre », c'est non seulement comme " moyen d'expression le plus authentique d'une existence particulière », comme « rempart » contre les « mœurs passe-partout », mais aussi parce qu'il renforce le statut du français. Insensible à l'argument voulant que patoiser freine l'apprentissage du français, Zermatten fait valoir que les « meilleurs élèves » seraient «ceux-là justement qui apprirent le français à l'école et non dans la famille et dans la rue », le français de la rue étant « vicié, entaché de toutes sortes d'erreurs ». Et à l'appui de cette thèse, il cite l'écrivain vaudois René Morax, selon qui les « lettres paysannes » venues d'Evolène (Valais) étaient « plus correctes et légères que les lettres citadines », puisque le berger qui parle à la maison sa « langue naturelle» (le patois) apprenait à l'école « la bonne grammaire ». Le FP, loin de nuire à la maîtrise du «langage de France », ne ferait que «l'enrichir de sa sève, de sa richesse concrète, de son penchant aux images ». On retrouve ici l'argument selon lequel une langue réputée colorée enrichira le français - associé à la rationalité.

Un autre élément du patriotisme linguistique des Romands est le rejet de l'influence de l'allemand, et le FP peut aussi jouer un rôle. Zermatten - l'un des rares à ne pas exclure la revitalisation du FP - s'en prend au journaliste Pierre Grellet, qui juge « illusoire de vouloir réimplanter le patois »- cause «irrémédiablement perdue » et désormais « article de musée ». Car si Grellet se réjouit que même les «fanatiques du patois » ne songent pas à « enseigner notre franco-provençal à des petits Lausannois », c'est en particulier parce que l'énergie investie dans le FP serait une « déperdition de force » et fragiliserait les Romands dans leur lutte contre «l'invasion germanique ». Or, Zermatten récuse l'idée que les régions patoisantes seraient « plus perméables au germanisme », et assure que la « poussée germanique » à la frontière des langues a été mieux contenue « dans les pays de langue romande » des cantons de Fribourg (La Roche) et du Valais (Anniviers, Hérens). Selon lui, ce n'est pas le français, mais le patois « qui refoula l'allemand sur la rive droite de la Sarine ». La disparition du FP serait non seulement « un immense appauvrissement », mais face à la menace de germanisation, le FP permettrait de mieux fixer «à leur coin de terre nos populations paysannes », fidèles à «l'accent de leur race». Pour montrer que le patois ne peut qu'épauler le français, il conclut: « pour bien servir la langue française que nous aimons, participons à la défense de notre langue maternelle, le franco-provençal ». 
On le comprend, la question du FP - même appelé à disparaître - est liée à celle du français régional et de l'utilisation de régionalismes peu compris dans d'autres pays francophones - sans oublier la question des emprunts à l'allemand. La hiérarchisation des langues place le français standard (parisien) en haut de la pyramide en termes de prestige, suivi du français régional (romand ou vaudois) et du FP. Lorsqu'un interlocuteur du journal (1943.12.14) entend légitimer l'utilisation du français régional ( il faut parler vaudois»), le journal, sous la plume de Jean Nicollier, rappelle le statut supérieur du français standard. Tout en affichant sa «tendresse » pour les « vieux mots du terroir », l'auteur précise que celle-ci ressemble à celle qu'on « voue à des objets anciens » (il cite bricelets) et refuse de trouver «prétentieuse » la langue de Voltaire. Citant Ramuz, qui classait les Vaudois «dans la catégorie des "[F]ranco-provençaux" », il conseille aux défenseurs du parler local de dépasser sa dimension vaudoise en combattant autant les «termes "provençaux" [sic] » (on retrouve ici l'ambiguité de la référence provençale) que les germanismes qui suscitent plus la vigilance. Pour montrer que l'énergie linguistique d'un bon Romand devrait aller à la défense du français standard, il s'applique à discréditer « notre défenseur des "Vaudoisismes" » en imaginant une invitation rédigée par un secrétaire municipal dans un français truffé de mots régionaux abscons, alors que le rôle d'une langue est de « maintenir des communications entre individus » et non d' élever entre eux des cloisons étanches»:

Le Conseil minicipâ a la joie de convoquer les habitants à une consulte publique dans la grande carrée communale. Les clédars (portes) seront ouverts contre les trois heures. Les arrivants sont priés de s'aguiller en ordre sur les bancs et de laisser les places du fin coutset libres pour les autorités. [italique et parenthèses dans l'original] (1943.12.14)

De façon similaire, le même journaliste reproche à Aldo Dami d'affirmer que le français standard, «importé en Suisse romande », serait une « langue en quelque sorte apprise » (contrairement au français de Suisse plus proche du FP), et de provoquer ainsi une «crise du français » (1951.12.19). Tout en concédant que la « fixité d'un idiome » n'existe pas et qu'il « découvre de la saveur à "câclon" » (le poêlon à fondue), tout en défendant l'idée d'un certain continuum entre niveaux de langue, il refuse de considérer le français francoprovençalisé de Suisse comme une langue autonome qui ferait des Romands des bilingues au prétexte qu'« à chacun de nous, il échappe fatalement des expressions et des tournures que nous ne saurions écrire », lorsque la langue occupe une « place intermédiaire » entre langue écrite et langue parlée ». Assumant un certain purisme, il exclut de « légitimer toutes les horreurs sous l'humble prétexte d'évolution » et invite chacun à mieux prendre soin du français.

Dans un autre texte sur le français régional (1951.4.3), le journaliste (J. Nr.) refuse la création d'un Office romand de la langue française (souhaitée par Camille Dudan, directeur de collège) et préfère aux « défenseurs parfois passionnés du parler local (ou régional) triomphant » ceux qui, « sans jeter le discrédit sur la langue et les expressions du cru, jugent que l'écrivain doit s'exprimer de façon à être compris sans effort à Paris comme à Lausanne, à Bruxelles comme à Lyon ». Sans nier l'importance historique du FP, il l'intègre à la mouvance du français plutôt que de l'en détacher. Rejetant tout séparatisme linguistique, il n'attribue qu'une place subalterne au FP, gardant intacte la pyramide 
symbolique, et se montre plus préoccupé de repousser les germanismes que de cultiver l'originalité des expressions vaudoises:

Certes, rien n'est plus français [nous soulignons] que les dialectes franco-provençaux dont nous sommes. Le « vaudois notre langue » a son prix à condition de demeurer à sa juste place: une place modeste, celle qu'aux côtés du pur français [italique dans l'original] digne de ce terme, le breton, le provençal, le belge ont le droit de revendiquer sans prétendre, toutefois, jusqu'à s'arroger le premier rang, le rang unique. [...] [Nous avons] le droit de saluer la couleur de vieux mots de chez nous à la saveur exquise. Mais notre devoir, lui, consiste à lutter contre nos pires ennemis: les germanismes amoureusement propagés [...]. [N]ous rendrons un plus réel service au français littéraire. (1951.4.3)

Le spectre de la germanisation est également brandi dans un texte sur une Suisse romande qui serait «menacée dans son intégrité linguistique »(1953.6.13). Claude Bodinier, journaliste fondateur de l'association suisse Défense du français, y évoque le déclin de l'universalisme du français qu'il met au compte des « poussées nationalistes » (il pense ici à celles des minoritaires, non des majoritaires) qui minent son autorité. Il ironise sur l'UNESCO qui promeut « les parlers indigènes au rang de langues de culture » et encourage « la traduction de Descartes dans l'idiome de Guagadogou [sic] ». Dans cette conception très colonialiste du français et de son prétendu universalisme (la référence à Rivarol apparaît également), il s'agit de frapper d'inexistence ou d'insignifiance les langues mineures, y compris le FP, subordonné à la défense du français - qui doit désormais présider seul à l'âme romande.

Cependant, malgré son statut subalterne, le FP est célébré en tant qu'il favoriserait le « bon bilinguisme » (celui qui exclut l'influence de l'allemand, réputée «fatale »). Comme le soulignait Zermatten, avoir le patois comme langue première permettrait d'apprendre à l'école un français plus pur et correct. Le patois, loin d'être un corps étranger, féconderait le français :

Il est un bon et un mauvais bilinguisme. Le «bon », c'est celui des patoisans [sic]. Ceux qui, chez nous, dans la Broye, en Valais, en Gruyère, parlent encore nos patois franco-provençaux, frappent justement par la clarté de leur élocution française, par la richesse de leur vocabulaire. C'est que le patois est une sève, et il faut être attentif à ne pas en effacer les traces savoureuses, que les pédants pourchassent à tort comme « provincialismes ». À cet égard, n’ayons pas honte d'être des bilingues sans le savoir - ou sans y penser. Et soutenons les efforts de ceux qui rassemblent patiemment les éléments de ce précieux patrimoine. (1953.6.13)

Quant au « mauvais bilinguisme, le bilinguisme proprement dit », il consiste, au lieu d'apprendre à fond un idiome puis un autre (FP puis français), à « apprendre deux idiomes à peu près simultanément, donc à penser selon deux mentalités, et appauvrir ses modes d'expression en cherchant inconsciemment les seuls qui aient des équivalents dans les deux langues ». Le remède face à cet ennemi désigné, l'allemand, est le « principe territorial - une terre, une langue » qui oblige « la main d'œuvre alémanique » immigrée en terre francophone à apprendre le français, mais aussi le refus 
d'une introduction trop prématurée de l'enseignement de l'allemand, car « la pénétration de l'allemand dans nos mœurs » risque d'entraîner l'« affaiblissement de la volonté de rester soi-même », voire la « disparition de la Suisse romande à plus ou moins brève échéance ».

Par cette distinction entre deux types de bilinguisme, Bodinier œuvre à la construction symbolique d'une communauté imaginée largement fondée sur l'unilinguisme. En dénonçant ceux qui, à l'extérieur de la Suisse, imaginent que les Suisses sont tous bilingues (français/allemand), il montre du reste qu'il ne prend pas très au sérieux le bilinguisme FP/français. Le bon bilinguisme qu'évoque l'auteur (et qui rappelle la diglossie) n'étant plus guère répandu (sauf sous forme de cohabitation entre français standard et français régional), c'est surtout son caractère inoffensif qui intéresse l'auteur. Face à l'accélération de la francisation de la Suisse romande, le FP peut être d'autant plus facilement valorisé - et utilisé comme alibi pour contrer le mauvais bilinguisme (germanisant) - qu'il est sur le déclin et fait l'objet d'une conservation patrimoniale, et non d'une protection comme langue vivante.

\section{Savoie et Vallée d'Aoste : quel parallèle entre les corpus vaudois et genevois?}

Nous pouvons déjà conclure que les Vaudois semblent s'être habitués à l'extinction prochaine de leur langue ancestrale, et que la construction d'une communauté imaginée (romande, mais surtout vaudoise) ne passe plus par la fidélité au patois, mais par un intérêt pour le français régional. Si on compare ce corpus vaudois avec un autre corpus, fondé sur les occurrences du terme franco-provençal dans le Journal de Genève (Meune, à paraître), il apparaît que la référence savoyarde est très peu présente dans la Gazette de Lausanne et qu'elle n'alimente guère la réflexion sur la construction d'une entité FP transnationale. Du côté vaudois, on semble davantage se concentrer sur la référence vaudoise que, du côté genevois, sur la référence genevoise. Dans le cas de Genève, la référence régionale semble plus propice à la construction d'une communauté transfrontalière, puisque le canton, presque enclavé en Savoie, a une histoire très liée à cette dernière, au point que le FP de Genève est parfois qualifié de savoyard - contrairement au FP vaudois.

En revanche, le Val d'Aoste occupe une place particulière dans les deux corpus. Si, dans le corpus genevois, la région italienne alimente la réflexion sur un domaine FP dépassant les frontières de la Suisse, c'est aussi le cas, dans une moindre mesure, dans le corpus vaudois. Ainsi, on lit que les Valaisans patoisants savent que leur patois « est exactement le même » au Val d'Aoste, où existerait cependant un «franco-provençal absolument pur » (1984.10.20). Cette dernière affirmation a d'autant moins de sens que le FP est fractionné et non normé, mais elle montre que les représentations de ce qu'est une vraie langue associent souvent celle-ci à un lieu dépositaire de l'authenticité. 
La Val d'Aoste permet aussi d'évoquer des réalités sociolinguistiques dépassant le cadre de la Suisse romande. Dans «Val d’Aoste: un génocide culturel » (1974.8.9), Laurent Rebeaud, journaliste et homme politique, souligne que les nouveaux tunnels (Mont-Blanc et Grand-Saint-Bernard) n'ont guère contribué à faire connaître le Val d'Aoste. Pourtant, il présente les Suisses romands comme " "sensibilisés" à la question des minorités linguistiques », notamment « à cause du Jura ». Ce dernier, malgré l'ancienne prégnance du «colonialisme » des germanophones bernois, aurait du reste des " couleurs paradisiaques », dès lors qu'on le compare à la situation valdôtaine. Alors qu'en 1860, « toute la vallée parlait français, ou le patois franco-provençal du lieu », l'immigration d'italophones et l'italianisation de la culture, imposées par le fascisme et à peine modifiées - sinon symboliquement - avec la nouvelle démocratie en 1945, auraient amené les autonomistes à réclamer l'application d'un statut jadis dénoncé comme inacceptable (s'agissant du nombre d'heures d'enseignement en français). Si le statut francophone du Val d'Aoste est traité sur un ton alarmiste, le FP est quant à lui présenté comme une langue de substitution susceptible de combattre l'italianisation et de retarder le " génocide culturel [à] deux heures de voiture de chez nous ». Comme face à l'allemand en Suisse, le FP se voit paré des vertus de la résistance.

Et dans une lettre qui fait du Val d'Aoste une " patrie oubliée de la francophonie » (1974.8.15), André Amiguet - traducteur de profession - évoque une « région du franco-provençal » dont les points extrêmes seraient Besançon, Grenoble et Aoste, une communauté «marquée du sceau de la même noblesse montagnarde » et "ennoblie par l'âme d'une même civilisation alpestre ». Le texte mentionne certes la Franche-Comté et la Savoie, mais il souligne particulièrement les liens entre Suisse romande et Val d'Aoste, «nos deux régions de même langue - nous n'insisterons jamais assez là-dessus ». L'auteur ajoute que « le vrai Valdotain parle trois langues »: le patois d'origine FP, le français et (par nécessité) l'italien. Et les Suisses, qui n'ont pas eu à se battre pour conserver le français, devraient selon lui au moins prendre à cœur la défense du FP. La pression assimilatrice en vigueur en Vallée d’Aoste devrait « nous encourager, nous qui avons négligé notre patois, à épauler nos frères de langue ", écrit l'auteur, comme s'il s'agissait, à défaut de conserver le rôle du français dans la culture valdôtaine, de sauver le FP pour faire honneur (rétrospectivement et indirectement) au FP de Suisse. 


\section{Conclusion}

Le corpus que nous venons d'étudier atteste qu'en un siècle, la Gazette de Lausanne a jeté les fondations de la construction par l'imaginaire d'une zone FP transfrontalière, mais que l'édifice reste inachevé. Alors que le Journal de Genève avait fait une référence à l'(h)arpitan dès 1975 (Meune, à paraître), on ne trouve dans la Gazette aucune trace de la future Arpitanie, dont les contours ont commencé à se dessiner plus précisément à la fin des années 2000, comme en témoignent divers journaux romands. Les arpitanistes sont des néo-locuteurs de FP qui, très présents sur Internet (v. Meune, 2014), cherchent à remplacer l'hybride franco(-)provençal par le terme arpitan, réputé plus attrayant, dans l'espoir de faciliter l'identification avec le domaine FP (dont nous avons vu qu'elle ne va pas de soi) ainsi que son unification, en particulier en promouvant une orthographe commune, dite ORB, pour « Orthographe de référence B » (Stich, 2003). On peut émettre l'hypothèse qu'avec la disparition des derniers locuteurs natifs de FP en Suisse - et la concentration de la presse romande -, les journaux insisteront moins sur les contextes cantonaux respectifs. Le FP, devenu enfin langue autonome après avoir été perçu comme un élément de continuum ou comme la somme de variétés dont le nombre masquait la parenté, continuera d'évoluer. Malgré l'attachement aux histoires cantonales et la prégnance des communautés symboliques romande et suisse, ceux qui s'intéresseront au FP (ou à l'arpitan) seront de plus en plus incités, s'ils souhaitent que le FP traverse encore ce siècle, à penser son avenir en termes transnationaux - sinon protonationaux. 


\section{Références}

Anderson, Benedict (1991), Imagined communities : reflections on the origin and spread of natonalism, London, Verso. [1'éd., 1983.]

Ascoli, Graziadio Isaia (1878), « Schizzi franco-provenzali », Archivio glottologico italiano, n 3, p. 61120.

Bert, Michel, Costa, James et Jean-Baptiste Martin (dir.) (2009), Étude FORA : francoprovençal et occitan en Rhône-Alpes, Lyon, Institut Pierre Gardette, Université catholique.

Blanchet, Philippe (2016), Discriminations : combattre la glottophobie, Paris, Textuel.

D’Oingt, Marguerite (2012), Expériences mystiques et récits édifiants: textes rédigés en francoprovençal et en latin par une moniale du XIII' siècle, édition bilingue (francoprovençal/latin-français), Lyon, EMCC.

Gadient, Irma (2012), «Ein lächerlicher Sprachenmischmasch : Dialekte und Sprach-vorstellungen im Kanton Freiburg Ende des 19. Jahrhunderts ", Revue transatlantique d'études suisses, n 2 (Le francoprovençal en Suisse : genèse, déclin, revitalisation, sous la dir. de Marinette Matthey et Manuel Meune), p. 33-56.

Kristol, André (2016), « Francoprovençal », dans Adam Ledgeway et Martin Maiden (dir.), The Oxford guide to the romance languages, Oxford: Oxford University Press, p. 350-362

Gauchat, Louis et al. (dir.) (1924), Glossaire des patois de la Suisse romande, Neuchâtel/Genève.

Hobsbawm, Eric (1992), Nations et nationalismes depuis 1780 : programme, mythe, réalité, Paris, Gallimard.

Matthey, Marinette (2012), « Quand ça a besoin de place, ça pousse : discours familial intergénérationnel sur la (non-)transmission du patois d'Evolène », Revue transatlantique d'études suisses, n 2 (Le francoprovençal en Suisse : genèse, déclin, revitalisation, sous la dir. de Marinette Matthey et Manuel Meune), p. 77-88, disponible sur Ilm.umontreal.ca/recherché/publications.html [Page consultée le 11 décembre 2016.]

Matthey, Marinette et Manuel Meune (dir.) (2012), Le francoprovençal en Suisse : genèse, déclin, revitalisation, numéro thématique de la Revue transatlantique d'études suisses, vol. 2, n 1 , disponible sur Ilm.umontreal.ca/recherché/publications.html [Page consultée le 11 décembre 2016.]

Meune, Manuel (2012a), « Parler patois ou de patois ? Locuteurs gruériens et néolocuteurs vaudois: le discours sur le francoprovençal dans les associations de patoisants », Revue transatlantique d'études suisses, $n^{\circ} 2$ (Le francoprovençal en Suisse : genèse, déclin, revitalisation, sous la dir. de Marinette Matthey et Manuel Meune), p. 57-75, disponible sur Ilm.umontreal.ca/recherché/publications.html [Page consultée le 11 décembre 2016.] 
Meune, Manuel (2012b), Pratiques et représentations des langues chez les locuteurs du francoprovençal fribourgeois : enquête sur la Société des patoisants de la Gruyère, Université de Montréal, disponible sur llm.umontreal.ca/recherché/publications.html [Page consultée le 11 décembre 2016.]

Meune, Manuel (2014), «Enjeu local et défi transnational, terroirs patoisants et exterritorialité arpitane : le francoprovençal à l'heure de Wikipédia », dans Didier Lassalle et Dirk Weissman (dir.), Ex(tra)territorial : Assessing Territory in Literature, Culture and Languages, Amsterdam/New York, Rodopi, p. 261-284.

Meune, Manuel (2015), «Lieu sans langue et langue sans lieu? Du Pays de Vaud au Valais, de Chappaz à Chessex : le fait francoprovençal dans la littérature de Suisse romande », dans Boris Czerny et Éric Leroy du Cardonnoy (dir.), Lieu(x) d'écriture et écriture de lieu(x) : topographie du réel à l'imaginaire, Caen, Presses universitaires de Caen, p. 147-159.

Meune, Manuel (À paraître), « Du patois à l'harpetan, entre (petite) patrie et nation imaginée : le discours sur le francoprovençal dans le Journal de Genève (1826-1998) », International Journal of the Sociology of Language.

Stich, Dominique (2003), Dictionnaire francoprovençal/français - français/ francoprovençal, Thononles-Bains, Le Carré.

Tuaillon, Gaston (2001), La littérature en francoprovençal avant 1700, Grenoble, Ellug.

Tuaillon, Gaston (1983), Le francoprovençal : progrès d'une définition, Saint-Nicolas, Centre d'études francoprovençales René Willien. 